\title{
Zentralvorstandssitzung vom 28. Juni 2012
}

Forum medizinische Register - Im Rahmen des Ende Juni abgeschlossenen Projekts «Forum medizinische Register Schweiz» wurde eine Online-Plattform gebildet, welche die gesammelten Informationen über die Register zur Verfügung stellt und die Vernetzung unter den Registerbetreibenden fördert. Damit stellt die FMH eine in der Schweiz zuvor nicht vorhandene Transparenz über die medizinischen Register her.

Ersatzwahl für Kommission - Die Schweizerische Kommission für Berufsentwicklung und Qualität ist für die Überwachung und Anpassung der Ausbildungsgrundlagen für MPA zuständig. Nach dem Rücktritt von Dr. med. Emil Schalch hat der Zentralvorstand (ZV) Dr. med. Katrin Hubschmid als offizielle FMH-Vertreterin in diesem Gremium gewählt.

Initiative «Schutz vor Passivrauchen» - Die Initiative «Schutz vor Passivrauchen», über die am 23. September abgestimmt wird, fordert ein Rauchverbot für alle Arbeitsplätze in Innenräumen, da die heutige Regelung trotz Bundesgesetz nicht ausreiche. Die FMH engagiert sich in der Abstimmungskampagne und wird die kantonalen Ärztegesellschaften sowie die betroffenen Fachgesellschaften zur Unterstützung aufrufen.
Film zum elektronischen Patientendossier - Das Koordinationsorgan eHealth des Bundes plant, zu Kommunikationszwecken einen Film über das elek tronische Patientendossier herzustellen. Der ZV will das Logo nur vergeben, wenn die FMH Einfluss auf den Inhalt des Films hat und diesen vor der Fertigstellung gutheissen kann.

Breitere Patentrechte - Aufgrund einer Praxisänderung der Beschwerdekammer des europäischen Patentamtes erhalten Patentinhaber mehr Rechte: Künftig kann auch ein bestimmtes Dosierungsschema eines Arzneimittels patentiert werden. Dadurch könnten aber Patentverletzungsklagen auf Ärzte zukommen, die Generika im «off label use» verschreiben oder verabreichen. Hier ortet der ZV Handlungsbedarf für die Schweiz. Er wird im August nach Prüfung eines von der Industrie zu erarbeitenden Lösungsvorschlags für ein Gentlemen's Agreement definitiv Stellung nehmen.

Ausschaffungen im Asylbereich - Der ZV ist mit dem Entwurf einverstanden, den Bundesrätin Simonetta Sommaruga zur Revision von Asylverordnungen in Auftrag gegeben hat. Dieser soll aber die Anpassung enthalten, die Ausschaffungen so $\mathrm{zu}$ gestalten, dass Ärztinnen und Ärzte ihre Aufgabe vollumfänglich erfüllen können, etwa mit der laufenden Überwachung des Gesundheitszustands des Auszuschaffenden.

\section{Wissen, was läuft.} Das News-Paket der FMH.

\author{
Schweizerische Ärztezeitung, Today's Press, \\ politoscope. Für Mitglieder kostenlos.
}

